\title{
Josephson-Junction Qubits with Controlled Couplings
}

\author{
Yuriy Makhlin*,†, Gerd Schön*, and Alexander Shnirman ${ }^{\ddagger}$ \\ *Institut für Theoretische Festkörperphysik, Universität Karlsruhe, D-76128 Karlsruhe, Germany. \\ ${ }^{\dagger}$ Landau Institute for Theoretical Physics, Kosygin St. 2, 117940 Moscow, Russia. \\ ${ }^{\ddagger}$ Department of Physics, University of Illinois at Urbana-Champaign, Urbana, IL 61801-3080, U.S.A.
}

Low-capacitance Josephson junctions, where Cooper pairs tunnel coherently while Coulomb blockade effects allow the control of the total charge, provide physical realizations of quantum bits (qubits), with logical states differing by one Cooper-pair charge on an island. The single- and two-bit operations required for quantum computation can be performed by applying a sequence of gate voltages. A basic design, described earlier [1], is sufficient to demonstrate the principles, but requires a high precision time control, and residual two-bit interactions introduce errors. Here we suggest a new nano-electronic design, close to ideal, where the Josephson junctions are replaced by controllable SQUIDs. This relaxes the requirements on the time control and system parameters substantially, and the two-bit coupling can be switched exactly between zero and a non-zero value for arbitrary pairs. The phase coherence time is sufficiently long to allow a series of operations.

A quantum computer can perform certain tasks which no classical computer is able to do in acceptable times [2 25]. It is composed of a (large) number of coupled two-state quantum systems forming qubits; the computation is the quantum-coherent time evolution of the state of the system described by unitary transformations which are controlled by the program. Elementary steps are (i) the preparation of the initial state of the qubits, (ii) single-bit operations (gates), i.e. unitary transformation of individual qubit states, triggered by a modification of the corresponding one-qubit Hamiltonian for some period of time, (iii) two-bit gates, which require controlled inter-qubit couplings, and (iv) the measurement of the final quantum state of the system. The phase coherence time has to be long enough to allow a large number of these coherent processes. Ideally, in the idle period between the operations the Hamiltonian of the system is zero to avoid further time evolution of the states.

Several physical realizations of quantum information systems have been suggested. Ions in a trap, manipulated by laser irradiation are the best studied system. However, alternatives need to be explored, in particular those which are more easily embedded in an electronic circuit as well as scaled up to large numbers of qubits. From this point of view mesoscopic and nano-electronic devices appear particularly promising [1]6 9]. Normal-metal singleelectron devices are discussed in connection with classical digital applications and, in fact, constitute the ultimate electronic memory [10]. However, their use for quantum computation is ruled out, since, due to the large number of electron states involved, different tunneling processes are incoherent. Ultra-small quantum dots with discrete levels are candidates for qubits, but their strong coupling to the environment renders their phase coherence time short. More promising are systems built from Josephson junctions, where the coherence of the superconducting state can be exploited. Quantum extension of elements based on a single-flux logic have been suggested, and attempts were made to observe coherent oscillations of flux quanta between degenerate states. Here we suggest to use low-capacitance Josephson junctions, where Cooper pairs tunnel coherently while Coulomb blockade effects allow the control of the total charge, encouraged by experiments which demonstrated the superposition of charge states [9,11. If biased near degeneracy these junctions constitute qubits with two logical states differing by one Cooper-pair charge on an island.

A simple design of Josephson junctions qubits and their coupling (reviewed below) has been suggested in Ref. [1]. Single-bit operations can be performed by controlling gate voltages applied to individual junctions, while two-bit gates can be implemented by tuning the selected qubits to resonance. The dephasing time has been estimated to be large compared to elementary operation times. To read out the quantum state a dissipative normal-metal single-electron transistor should be coupled to the qubit [12]. The low number of junctions and control voltages of the simple design should simplify an experimental realization. Drawbacks are the continuing time evolution of the states also during idle periods, which necessitates a high precision of the time control, as well as intrinsic errors introduced by nonvanishing twobit couplings even if the qubits are out of resonance. In this article we suggest an improved design, still based on nano-scale Josephson junction technology, which is close to ideal. The crucial step is the replacement of the Josephson junctions by SQUIDs which can be controlled by external magnetic fluxes. This allows us to switch the Josephson couplings between zero and non-zero values. It substantially reduces the requirements on the time control and provides a complete control of two-bit couplings.

An ideal model: To fix ideas we present a model of an ideal quantum computer with Hamiltonian

$$
H=-\sum_{i=1}^{N}\left[E_{z}^{i}(t) \hat{\sigma}_{z}^{i}+E_{x}^{i}(t) \hat{\sigma}_{x}^{i}\right]+\sum_{i \neq j} A^{i j}(t) \hat{\sigma}_{+}^{i} \hat{\sigma}_{-}^{j} .
$$


A spin notation is used for the qubits with Pauli matrices $\hat{\sigma}_{z}, \hat{\sigma}_{x}, \hat{\sigma}_{ \pm}=\left(\hat{\sigma}_{x} \pm i \hat{\sigma}_{y}\right) / 2$. Ideally, each energy $E_{z}^{i}(t), E_{x}^{i}(t)$ and the (real symmetric) couplings $A^{i j}(t)$ can be switched separately for controlled times between zero and finite values, $E_{z}^{i}, E_{x}^{i}$ and $A^{i j}$. We assumed that $E_{z}^{i}$ is the largest energy, suggesting the choice of basis states $\left|\uparrow_{i}\right\rangle$ and $\left|\downarrow_{i}\right\rangle$ aligned along the $z$-axis. Residual inelastic interactions, which destroy the coherence, and the measurement device, when turned on, should be accounted for by extra terms $H_{\text {res }}$ and $H_{\text {meas }}(t)$, respectively.

(i) For the system (11) the initial state can be prepared by turning on large values of $E_{z}^{i} \gg k_{\mathrm{B}} T, i=1, \ldots, N$ at low temperature for sufficient time (while $E_{x}^{i}(t)=A^{i j}(t)=$ $0)$, such that the residual interaction, $H_{\text {res }}$, relaxes all spins to the ground state, $|\uparrow \uparrow \uparrow \ldots\rangle$. Switching $E_{z}^{i}(t)$ back to zero leaves the system in a well defined state, and, since $H=0$, there is no further time evolution.

(ii) Single-bit operations are controlled by turning on one of the corresponding $E_{x}^{i}(t)$ for a time $\tau$. Hence, the spin $i$ evolves according to the unitary transformation

$$
U_{1 \mathrm{~b}}^{i}(\tau)=\exp \left(-i E_{x}^{i} \tau \hat{\sigma}_{x}^{i} / \hbar\right) .
$$

Depending on the time span, a $\pi / 2$ - or $\pi / 4$-rotation is performed, producing a spin flip or an equal-weight superposition of spin states. Switching on (a small) $E_{z}^{i}(t)$ for some time produces another needed operation: a phase shift between $\left|\uparrow_{i}\right\rangle$ and $\left|\downarrow_{i}\right\rangle$.

(iii) A two-bit operation on qubits $i$ and $j$ is achieved by turning on the corresponding $A^{i j}(t)$. In the basis $\left|\uparrow_{i} \uparrow_{j}\right\rangle$, $\left|\uparrow_{i} \downarrow_{j}\right\rangle,\left|\downarrow_{i} \uparrow_{j}\right\rangle,\left|\downarrow_{i} \downarrow_{j}\right\rangle$ the result is described by

$$
U_{2 \mathrm{~b}}^{i j}(\tau)=\left(\begin{array}{cccc}
1 & 0 & 0 & 0 \\
0 & \cos \left(A^{i j} \tau / \hbar\right) & i \sin \left(A^{i j} \tau / \hbar\right) & 0 \\
0 & i \sin \left(A^{i j} \tau / \hbar\right) & \cos \left(A^{i j} \tau / \hbar\right) & 0 \\
0 & 0 & 0 & 1
\end{array}\right) .
$$

For $A^{i j} \tau / \hbar=\pi / 2$ the result is a spin-swap operation, while $A^{i j} \tau / \hbar=\pi / 4$ yields a 'square root of swap'. The latter transforms the state $\left|\uparrow_{i} \downarrow_{j}\right\rangle$ into an entangled state $\frac{1}{\sqrt{2}}\left(\left|\uparrow_{i} \downarrow_{j}\right\rangle+i\left|\downarrow_{i} \uparrow_{j}\right\rangle\right)$. Combined with the single-bit operations it allows to perform a 'controlled-not' operation [6]. This combination provides a universal set of logic gates, sufficient for quantum computations [13.14].

(iv) The measurement process has to be discussed for specific realizations.

Simple Josephson qubits: Nano-scale Josephson junctions can serve as realizations of qubits. The simplest example is provided by the superconducting electron box [1] shown in Fig. 1a). The relevant conjugate variables are the charge $Q=2 n e$ on the island (where $n$ is the number of Cooper pairs) and the phase difference $\gamma$ across the junction. If normal electron tunneling is suppressed by the superconducting energy gap and only 'even-parity' states are involved [15], the circuit dynamics is described by the Hamiltonian

$$
H=\frac{\left(Q-C V_{\mathrm{x}}\right)^{2}}{2\left(C+C_{\mathrm{J}}\right)}-E_{\mathrm{J}} \cos \gamma ; Q=\frac{\hbar}{i} \frac{\partial}{\partial(\hbar \gamma / 2 e)} .
$$

For the junctions considered, the charging energy with scale $E_{C} \equiv e^{2} / 2\left(C+C_{\mathrm{J}}\right)$ dominates over the Josephson coupling $E_{\mathrm{J}}$. It is plotted in Fig. 2 as a function of the external voltage $V_{\mathrm{x}}$ for different island charges $n$. In equilibrium at $k_{\mathrm{B}} T \ll E_{C}$, the system is in the state corresponding to the lowest parabola. However, near the voltages $V_{\mathrm{deg}}=(2 n+1) e / C$ the states $n$ and $n+1$ are degenerate, and the Josephson coupling mixes them strongly. Here, the reduced two-state Hamiltonian in a basis of the charge states $|\uparrow\rangle=|n\rangle$ and $|\downarrow\rangle=|n+1\rangle$ is

$$
H=E_{\mathrm{ch}}\left(V_{\mathrm{x}}\right) \hat{\sigma}_{z}-\frac{E_{\mathrm{J}}}{2} \hat{\sigma}_{x},
$$

where $E_{\mathrm{ch}}\left(V_{\mathrm{x}}\right)=\frac{C_{\mathrm{qb}}}{C_{\mathrm{J}}} e\left(V_{\mathrm{x}}-V_{\mathrm{deg}}\right)$, and the capacitance of the qubit in the circuit is $C_{\mathrm{qb}}^{-1}=C_{\mathrm{J}}^{-1}+C^{-1}$.
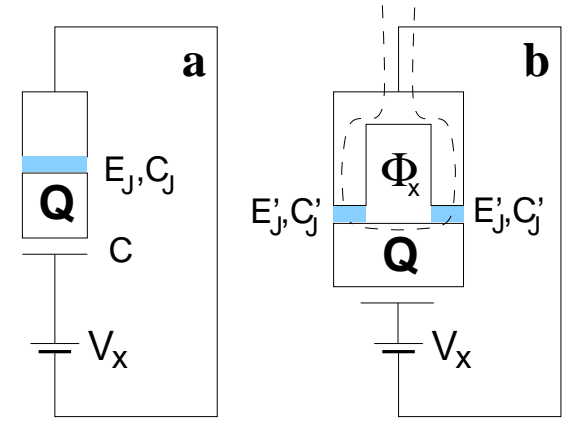

FIG. 1. Josephson junction qubits. a) A simple realization of a qubit is provided by the superconducting electron box. The important degree of freedom is the Cooper pair charge $Q=2 e n$ on the island between gate capacitor $C$ and Josephson junction (grey area) with capacitance $C_{\mathrm{J}}$ and Josephson coupling energy $E_{\mathrm{J}}$.

b) The improved design of the qubit. The island is coupled to the circuit via two Josephson junctions with parameters $C_{\mathrm{J}}^{\prime}$ and $E_{\mathrm{J}}^{\prime}$. This dc-SQUID can be tuned by the external flux which is controlled by the current through the inductor loop (dashed line). The setup allows switching the effective Josephson coupling to zero.

On the way towards the ideal model (11) we achieved a tunable $E_{z}^{i}(t)$, however, the Josephson coupling is fixed $E_{x}^{i}(t)=E_{\mathrm{J}} / 2$. Still single-bit operations can be performed by controlling the bias voltage $V_{\mathrm{x}}$ [1]. Furthermore, when the qubits are connected in parallel to a joint inductor (similar as in Fig. 3), the common $L C$-oscillator mode provides a two-bit coupling with weak, but constant $A^{i j} \sim \frac{C^{2}}{C_{\mathrm{J}}^{2}} \frac{E_{\mathrm{J}}^{2} L}{\Phi_{0}^{2}}$. This coupling provides a two-bit gate if two qubits, $i$ and $j$, are brought into resonance by 
biasing them with the same gate voltage $V_{\mathrm{x} i}=V_{\mathrm{x} j}$. Out of resonance it is only a weak perturbation.

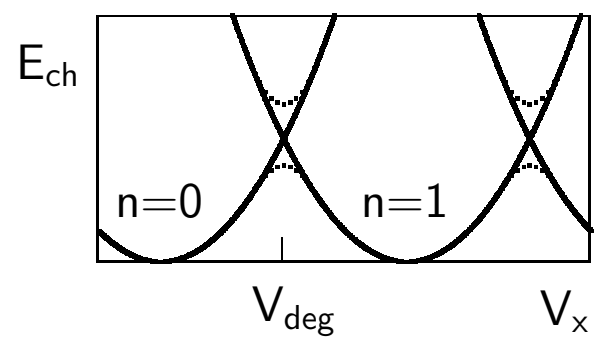

FIG. 2. Spectrum of a superconducting electron box. The charging energy of the superconducting electron box is shown (solid lines) as a function of the applied gate voltage $V_{\mathrm{x}}$ for different numbers of Cooper pair charges $n$ on the island. Near degeneracy points the weaker Josephson coupling energy mixes the charge states and modifies the energy of the eigenstates (dotted line). In this regime the system effectively reduces to a 2-state quantum system.

The external voltage source is part of a dissipative circuit with effective resistance $R_{V}$. This introduces fluctuations and destroys the phase coherence. Following Refs. [16,17 we can estimate the corresponding decoherence time. At the degeneracy point it is

$$
\tau_{V}=\frac{1}{4 \pi} \frac{R_{\mathrm{K}}}{R_{V}}\left(\frac{C_{\mathrm{J}}}{C_{\mathrm{qb}}}\right)^{2} \frac{\hbar}{E_{\mathrm{J}}} \tanh \left(\frac{E_{\mathrm{J}}}{2 k_{\mathrm{B}} T}\right) .
$$

The ratio of the quantum resistance $R_{\mathrm{K}}=h / e^{2} \approx 26 \mathrm{k} \Omega$ and $R_{V}$ determines the strength of the fluctuations. Furthermore, a small gate capacitance $C \ll C_{\mathrm{J}}$ helps decoupling the qubit from the environment. Both should be optimized to provide a phase coherence time much longer than typical operation times $\hbar / E_{\mathrm{J}}$.

A problem with the simple design is that the eigenstates of the Hamiltonian (5) are non-degenerate at all voltages. Therefore, the relative phase of two eigenstates evolves in time even during idle periods. We can still store quantum information in the qubit, as becomes apparent after a transformation to the interaction representation. This introduces, however, an explicit time dependence in the operators with nontrivial consequences for the unitary transformations. Their result does not only depend on the time span $\tau$ of the operations but also on the time $t_{0}$ when they start. As a consequence the time elapsed since the beginning of the computation should be controlled with high accuracy, determined by the spacing between the two eigenvalues of $H\left(V_{0}\right)$. A second problem of the simple design is the non-vanishing two-bit coupling even out of resonance. It introduces an error in the computation. The design discussed below overcomes both these problems.

Josephson qubit with SQUID-controlled coupling: The crucial step towards the ideal model (11) is to make the Josephson coupling tunable. This is achieved by the design shown in Fig. 1b), where each Josephson junction is replaced by a dc-SQUID threaded by the flux $\Phi$. The SQUID is biased by an external flux $\Phi_{\mathrm{x}}$, which is coupled into the system through an inductor loop. The energy of this element is

$$
\begin{aligned}
& E_{\mathrm{SQUID}}=\frac{1}{2 L_{\Phi}}\left(\Phi-\Phi_{\mathrm{x}}\right)^{2}-2 E_{\mathrm{J}}^{\prime} \cos \gamma \cos \left(\frac{\pi \Phi}{\Phi_{0}}\right) \\
& +C_{\mathrm{J}}^{\prime}\left(\frac{\hbar^{2}}{4 e^{2}} \dot{\gamma}^{2}+\frac{1}{4} \dot{\Phi}^{2}\right),
\end{aligned}
$$

where $\Phi_{0}=h /(2 e)$. The phase difference across the element $\gamma$ and flux $\Phi$ are dynamical variables. If the self-inductance $L_{\Phi}$ of the loop is low 15, $\Phi_{0}^{2} / L_{\Phi} \gg$ $(2 \pi)^{2} E_{\mathrm{J}}^{\prime}, e^{2} / C_{\mathrm{J}}^{\prime}$, fluctuations of the flux around $\Phi_{\mathrm{x}}$ are weak. Furthermore, if the frequency of flux oscillations $\omega_{\Phi}=\left(L_{\Phi} C_{\mathrm{J}}^{\prime} / 2\right)^{-1 / 2}$ is large, $\hbar \omega_{\Phi} \gg E_{\mathrm{J}}^{\prime}, E_{\mathrm{ch}}, k_{\mathrm{B}} T$, the $\Phi$-degree of freedom is in the ground state. In this case the SQUID-controlled qubit is described by a Hamiltonian of the form (4) with potential energy

$$
-2 E_{\mathrm{J}}^{\prime} \cos \left(\pi \Phi_{\mathrm{x}} / \Phi_{0}\right) \cos \gamma
$$

and effective junction capacitance $C_{\mathrm{J}}=2 C_{\mathrm{J}}^{\prime}$. I.e., the effective Josephson coupling $E_{\mathrm{J}}\left(\Phi_{\mathrm{x}}\right)=2 E_{\mathrm{J}}^{\prime} \cos \left(\pi \Phi_{\mathrm{x}} / \Phi_{0}\right)$ is tunable by the external flux $\Phi_{\mathrm{x}}$ between $2 E_{\mathrm{J}}^{\prime}$ and zero.

We note that the SQUID-controlled qubit is described by the first two terms of the ideal Hamiltonian (11), with $z$ - and $x$-components controlled independently by the gate voltage and the the flux. In the idle state we keep $V_{\mathrm{x}}=V_{\mathrm{deg}}$ and $\Phi_{\mathrm{x}}=\Phi_{0} / 2$ so that the Hamiltonian $H=0$. If we change one of them the new Hamiltonian generates rotations around $z$ - or $x$-axis, respectively, which are elementary one-qubit operations. Note, that with this design there is no need to control the total operation time $t_{0}$, while the voltage and $E_{\mathrm{J}}\left(\Phi_{\mathrm{x}}\right)$ can be optimized such that the duration of the manipulations $\tau$ is long enough to simplify time control and short enough to speed up the computation.

The circuit of the current source with resistance $R_{I}$, which couples the flux $\Phi_{\mathrm{x}}$ to the SQUID by the mutual inductance $M$, introduces fluctuations and may destroy the coherence of the qubit dynamics. At the degeneracy point the decoherence time is

$$
\tau_{I}=\frac{1}{\pi^{3}} \frac{R_{I}}{R_{\mathrm{K}}}\left(\frac{\Phi_{0}^{2}}{E_{\mathrm{J}}^{\prime} M}\right)^{2} \frac{\hbar}{k_{\mathrm{B}} T}
$$

This dephasing is slow if the current source is coupled weakly to the qubit (small $M$ ) and its resistance is high.

Controlled inter-qubit coupling: The controlled Josephson junctions allow us also to switch the two-qubit interaction for each pair of qubits, bringing us close to the ideal model (11). The simplest implementation of the coupling is to connect all $N$ qubits in parallel to each other to an inductor $L$ (Fig. 3). If the frequency of the 
$L C$-mode in the resulting circuit, $\omega_{L C}=\left(N C_{\mathrm{qb}} L\right)^{-1 / 2}$, is large $\hbar \omega_{L C} \gg E_{\mathrm{J}}, E_{\mathrm{ch}}, k_{\mathrm{B}} T$ the fast oscillations produce an effective coupling of the qubit dynamics [1]

$$
H_{\mathrm{int}}=\sum_{i<j} \frac{E_{\mathrm{J}}^{i} E_{\mathrm{J}}^{j}}{E_{L}} \hat{\sigma}_{y}^{i} \hat{\sigma}_{y}^{j},
$$

where $E_{L}=\frac{\Phi_{0}^{2}}{\pi^{2} L}\left(\frac{C_{\mathrm{J}}}{C_{\mathrm{qb}}}\right)^{2}$, and $E_{\mathrm{J}}^{i}=E_{\mathrm{J}}\left(\Phi_{\mathrm{x} i}\right)$ are the effective Josephson energies of the qubits, controlled by the external fluxes. The coupling energy (10) can easily be understood as the magnetic energy of the current in the inductor, where the current is the sum of contributions from all qubits $I^{i} \propto E_{\mathrm{J}}^{i} \hat{\sigma}_{y}^{i}$.

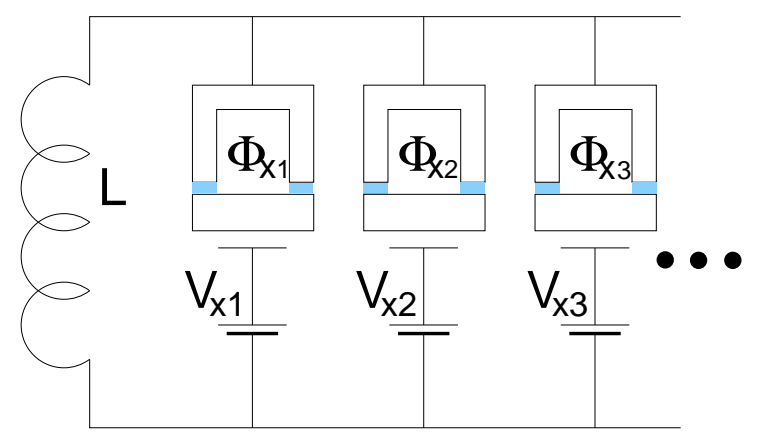

FIG. 3. Design of a quantum computer. The coupling of the qubits is provided by the $L C$-oscillator mode in circuit shown. Note that the system can be scaled to large numbers of qubits. In the idle state all effective Josephson couplings are tuned to zero and the voltages are chosen such that the charge states are degenerate. Single-bit operations are achieved by changing the gate voltage or flux of one qubit at a time. Two-bit operations between any two qubits are triggered by turning on the corresponding two Josephson couplings.

Using this interaction we can perform all gate operations. In the idle state the interaction Hamiltonian (10) is zero since all the Josephson couplings are turned off (the charge $Q_{i}$ is conserved and the qubit does not contribute to the current through the inductor). The same is true during a one-qubit operation, as long as we perform one such an operation at a time: i.e. only one $E_{\mathrm{J}}^{i} \neq 0$. To perform a two-qubit operation with any given pair of qubits, say 1 and $2, E_{\mathrm{J}}^{1}$ and $E_{\mathrm{J}}^{2}$ are switched on simultaneously, yielding the total Hamiltonian

$$
H=-\frac{E_{\mathrm{J}}^{1}}{2} \hat{\sigma}_{x}^{1}-\frac{E_{\mathrm{J}}^{2}}{2} \hat{\sigma}_{x}^{2}+\frac{E_{\mathrm{J}}^{1} E_{\mathrm{J}}^{2}}{E_{L}} \hat{\sigma}_{y}^{1} \hat{\sigma}_{y}^{2} .
$$

While not identical to the form (11) also these two-bit operations, combined with the one-bit operations discussed above, provide a complete set of gates required for quantum computation [13].

Discussion and outlook: To demonstrate that the constraints on the circuit parameters can be met by available technology, we suggest a suitable set:

(i) We choose junctions with capacitance $C_{\mathrm{J}}=3$. $10^{-16} \mathrm{~F}$, corresponding to a charging energy (in temperature units) $E_{C} \sim 3 \mathrm{~K}$, and a smaller gate capacitance $C=3 \cdot 10^{-17} \mathrm{~F}$ to reduce the coupling to the environment. The superconducting gap has to be slightly larger $\Delta>E_{C}$. Thus at working temperature of order $T=50 \mathrm{mK}$ the initial thermalization is assured. We further choose $E_{\mathrm{J}}^{\prime}=50 \mathrm{mK}$, i.e. the time scale of one-qubit operations is $\tau_{\mathrm{op}}=\hbar / E_{\mathrm{J}} \sim 7 \cdot 10^{-11} \mathrm{~s}$. Fluctuations associated with the gate voltages (6), with resistance $R_{V} \sim 50 \Omega$, limit the coherence time to $\tau_{V} / \tau_{\text {op }} \sim 4000$ operations.

(ii) If the inductance of the SQUID loop is $L_{\Phi}=0.1 \mathrm{nH}$, fluctuations of the flux are weak, $\left\langle\delta \Phi^{2}\right\rangle^{1 / 2} \sim 0.08 \Phi_{0}$, and, as required, the excitation energy of the $\Phi$-degree of freedom $\hbar \omega_{\Phi} \sim 80 \mathrm{~K}$ is large compared to characteristic energy scales of the qubit. Compared to the voltage fluctuations, for reasonable values $M=1 \mathrm{nH}$ and $R_{I}=10^{2}$ $10^{6} \Omega$, the flux circuit has a weak dephasing effect.

(iii) To assure fast two-bit operations we choose the energy scale $E_{L}$ of the order of $10 E_{\mathrm{J}}$, which is achieved for $L \sim 3 \mu \mathrm{H}$. Since the energy of $L C$-oscillations $\hbar\left(N C_{\mathrm{qb}} L\right)^{-1 / 2}$ should be large compared to $k_{\mathrm{B}} T$ and $2 E_{\mathrm{J}}^{\prime}$, the number of qubits in the circuit is limited by $N_{\max } \sim 70$.

We add two technical remarks:

(i) Above we assumed a very small SQUID inductance $L$. For finite values fluctuations of the flux renormalize the energy (8). But still, by symmetry arguments, at $\Phi_{\mathrm{x}}=\Phi_{0} / 2$ the effective Josephson coupling is zero.

(ii) While the expression (10) is valid in leading order in an expansion in $E_{\mathrm{J}}^{i} / \hbar \omega_{L C}$, higher terms also vanish when the Josephson couplings are put to zero. Hence, the decoupling in the idle periods persists.

Further remarks on the design and manipulation of the system are in order:

(i) The two lowest states of the qubit are separated from higher states, which exist in the physical system, by the energies $E_{C}, \hbar \omega_{L C}, \hbar \omega_{\Phi}$. If, in addition, switching processes of $V_{\mathrm{x}}$ and $\Phi_{\mathrm{x}}$ are slow on the corresponding time scales, the requirements presented above also ensure that the higher states are not excited. Alternatively, instead of sudden switching, one can apply resonance ac-signals after changing the biases adiabatically to finite values.

(ii) In addition to the gate operations the resulting quantum state has to be read out. This can be accomplished by coupling a normal-state single-electron transistor capacitively to a qubit. The important aspect is that during the computation the transistor is kept in a zero current state and adds only to the total capacitance. When the transport voltage is turned on, the dissipative current in the transistor depends on the state of the qubit, and the phase coherence of the q-bit is destroyed. This quantum measurement process has been described explicitly in Ref. [12] by an analysis of the time-evolution of the 
density matrix of the coupled system.

(iii) The system presented here does not permit parallel operations on different qubits, which is an essential element of many powerful quantum algorithms. It can be achieved in principle by a more advanced design, making use, e.g., of further tunable SQUIDs decoupling different parts of the circuit. Such modifications, as well as further progress of nano-technology, will provide longer coherence times and allow scaling to larger numbers of qubits. We stress, however, that many aspects of quantum informations processing can initially be tested on simple circuits as proposed here.

To conclude, the realization of a nano-scale quantum computers based on controlled Josephson qubits is possible with current technology. In such a system fundamental features of macroscopic quantum-mechanical systems can be further explored.

[1] Shnirman, A., Schön, G., and Hermon, Z. Quantum manipulations of small Josephson junctions. Phys. Rev. Lett. 79, 2371 (1997).

[2] Lloyd, S. A potentially realizable quantum computer. Science 261, 1589 (1993).

[3] Bennett, C. H. Quantum information and computation. Physics Today 48 (10), 24 (1995).

[4] DiVincenzo, D. P. Quantum computation. Science 269, 255 (1995).

[5] Barenco, A. Quantum physics and computers. Contemp. Phys. 37, 375 (1996).

[6] Loss, D., DiVincenzo, D. P. Quantum computation with quantum dots. Phys. Rev. A 57, 120 (1998).

[7] Mooij, J. E. private communication.

[8] Averin, D. V. Adiabatic quantum computation with Cooper pairs. Solid State Commun. 105, 659 (1998).

[9] Bouchiat, V., Joyez, P., Esteve, D., and Devoret, M. to be published in Physica Scripta; V. Bouchiat, Ph. D. Thesis, Université Paris 6, (1997).

[10] Korotkov, A. N., Chen, R. H., and Likharev, K. K. Possible performance of capacitively coupled single-electron transistors in digital circuits. J. Appl. Phys. 78, 2520 (1995).

[11] Maassen v.d. Brink, A., Schön, G., and Geerligs, L. J. Combined single-electron and coherent-Cooper-pair tunneling in voltage-biased Josephson junctions. Phys. Rev. Lett. 67, 3030 (1991).

[12] Shnirman, A. and Schön, G. Quantum measurements performed with a single-electron transistor. Phys. Rev. $B$ 57, 15400 (1998).

[13] Lloyd, S. Almost Any Quantum Logic Gate is Universal. Phys. Rev. Lett. 75, 346 (1995).

[14] Barenco, A. et al. Elementary gates for quantum computation. Phys. Rev. A 52, 3457 (1995).

[15] see, e.g., Tinkham, M. Introduction to Superconductivity. McGraw-Hill, New York, 1996.
[16] Leggett, A. J. et al. Dynamics of the dissipative two-state system. Rev. Mod. Phys. 59, 1 (1987).

[17] Weiss, U. Quantum dissipative systems. World Scientific, Singapore, 1993.

Acknowledgments: We thank T. Beth, M. Devoret, D. P. DiVincenzo, E. Knill, K. K. Likharev, and J. E. Mooij for stimulating discussions.

Correspondence should be addressed to Y.M. (e-mail: makhlin@tfp.physik.uni-karlsruhe.de) 